\title{
Upper Bounds on Spontaneous Wave-Function Collapse Models Using Millikelvin-Cooled Nanocantilevers
}

\author{
A. Vinante, ${ }^{1,2, *}$ M. Bahrami, ${ }^{3,4}$ A. Bassi, ${ }^{3,4}$ O. Usenko, ${ }^{5}$ G. Wijts, ${ }^{5}$ and T. H. Oosterkamp ${ }^{5}$ \\ ${ }^{1}$ Istituto Nazionale di Fisica Nucleare (INFN), TIFPA, I-38123 Povo, Trento, Italy \\ ${ }^{2}$ Istituto di Fotonica e Nanotecnologie, CNR-Fondazione Bruno Kessler, I-38123 Povo, Trento, Italy \\ ${ }^{3}$ Department of Physics, University of Trieste, Strada Costiera 11, 34014 Trieste, Italy \\ ${ }^{4}$ Istituto Nazionale di Fisica Nucleare (INFN), Trieste Section, Via Valerio 2, 34127 Trieste, Italy \\ ${ }^{5}$ Leiden Institute of Physics, Leiden University, Post Office Box 9504, 2300 RA Leiden, The Netherlands \\ (Received 16 October 2015; revised manuscript received 12 January 2016; published 2 March 2016) \\ Collapse models predict a tiny violation of energy conservation, as a consequence of the spontaneous \\ collapse of the wave function. This property allows us to set experimental bounds on their parameters. We \\ consider an ultrasoft magnetically tipped nanocantilever cooled to millikelvin temperature. The thermal \\ noise of the cantilever fundamental mode has been accurately estimated in the range $0.03-1 \mathrm{~K}$, and any \\ other excess noise is found to be negligible within the experimental uncertainty. From the measured data \\ and the cantilever geometry, we estimate the upper bound on the continuous spontaneous localization \\ collapse rate in a wide range of the correlation length $r_{C}$. Our upper bound improves significantly previous \\ constraints for $r_{C}>10^{-6} \mathrm{~m}$, and partially excludes the enhanced collapse rate suggested by Adler. We \\ discuss future improvements.
}

DOI: 10.1103/PhysRevLett.116.090402

Spontaneous wave function collapse models [1-4] have been proposed to reconcile the linear and deterministic evolution of quantum mechanics with the nonlinear and stochastic character of the measurement process. According to such phenomenological models, random collapses occur spontaneously in any material system, leading to a spatial localization of the wave function. The collapse rate scales with the size (number of constituents) of the system, leading to rapid localization of any macroscopic system, while giving no measurable effect at the microscopic level, where conventional quantum mechanics is recovered.

Here we consider the mass-proportional version of the continuous spontaneous localization (CSL) model [2], the most widely studied one, originally introduced as a refinement of the Ghirardi-Rimini-Weber (GRW) model [1]. CSL is characterized by two phenomenological constants, a collapse rate $\lambda$, and a characteristic length $r_{C}$, which characterize, respectively, the intensity and the spatial resolution of the spontaneous collapse. The standard conservative values suggested for the CSL parameters are $\lambda \simeq 10^{-17} \mathrm{~s}^{-1}$ and $r_{C}=10^{-7} \mathrm{~m}[1,2]$. A strongly enhanced value for $\lambda$ has been suggested by Adler [5], motivated by the requirement of making the wave-function collapse effective at the level of latent image formation in photographic process. The values suggested by Adler are $\sim 10^{9 \pm 2}$ times larger than standard values at $r_{C}=10^{-7} \mathrm{~m}$, and $\sim 10^{11 \pm 2}$ times larger at $r_{C}=10^{-6} \mathrm{~m}$.

The direct effect of collapse models like CSL is to destroy quantum superpositions, resulting in a loss of coherence in interferometric tests with matter-wave [6-8] or mechanical resonators [9-11]. Recently, noninterferometric tests have been proposed, which promise to set stronger bounds on these models [12-19]. Among such tests, the measurement of heating effects in mechanical systems, a byproduct of the collapse process, seems particularly promising [15-18]. Here, we demonstrate for the first time this method, by accurately measuring the mean energy of a nanocantilever in thermal equilibrium at millikelvin temperatures. We infer an experimental upper bound on $\lambda$, which is 2 orders of magnitude stronger than that set by matter-wave interferometry [20-22] for $r_{C}=10^{-7} \mathrm{~m}$, and the strongest one to date for $r_{C}>10^{-6} \mathrm{~m}$.

Theoretical model.-The detection of CSL-induced heating in realistic optomechanical systems has been extensively discussed in the recent literature [15-19]. Here we summarize the main steps. At the density matrix level, the CSL model is described by a Lindblad type of master equation for the density matrix $\rho$, with the Lindblad term (projected on the $N$-particle subspace of the Fock space, in momentum representation) given by

$$
\begin{aligned}
\mathcal{L}_{\mathrm{CSL}}[\hat{\rho}(t)]= & -\frac{(4 \pi)^{\frac{3}{2}} \lambda r_{C}^{3}}{2 m_{0}^{2}} \sum_{i, j=1}^{N} m_{i} m_{j} \int \frac{d^{3} \mathbf{k}}{(2 \pi)^{3}} \\
& \times e^{-r_{C}^{2} \mathbf{k}^{2}}\left[e^{i \mathbf{k} \cdot \hat{\mathbf{x}}_{i}},\left[e^{-i \mathbf{k} \cdot \hat{\mathbf{x}}_{j}}, \hat{\rho}(t)\right]\right],
\end{aligned}
$$

where $i$ and $j$ label the number of particles, $m_{i}$ and $\hat{\mathbf{x}}_{i}$ are the mass and position operator of particle $i$, and $m_{0}=1 \mathrm{amu}$.

We consider a mechanical resonator in equilibrium with a phononic thermal bath at temperature $T$. When the spatial motion of the resonator is smaller than $r_{C}$, as in our experiment $\left(|\Delta x| \sim 10^{-9} \mathrm{~m}\right)$, Eq. (1) can be Taylor 
expanded. In the case of a rigid body, the Lindblad effect on the center-of-mass motion becomes $[15,16]$

$$
\mathcal{L}_{\mathrm{CSL}}\left[\hat{\rho}_{\mathrm{CM}}(t)\right]=-\frac{1}{2} \eta\left[\hat{q},\left[\hat{q}, \hat{\rho}_{\mathrm{CM}}(t)\right]\right],
$$

$\hat{q}$ being the position operator of the center of mass, and

$$
\eta=\frac{(4 \pi)^{\frac{3}{2}} \lambda r_{C}^{3}}{m_{0}^{2}} \int \frac{d^{3} \mathbf{k}}{(2 \pi)^{3}} k_{z}^{2} e^{-\mathbf{k}^{2} r_{C}^{2}}|\tilde{\varrho}(\mathbf{k})|^{2},
$$

with $\mathbf{k}=\left(k_{x}, k_{y}, k_{z}\right), \tilde{\varrho}(\mathbf{k})=\int d^{3} \mathbf{x} e^{i \mathbf{k} \cdot \mathbf{r}} \varrho(\mathbf{r})$, and $\varrho(\mathbf{r})$ the mass density of the oscillator. The motion is only in one spatial direction which we set as the $z$ axis. The effect of the Lindblad term in Eq. (2) can be mimicked by adding the stochastic potential $V(t)=-\hbar w_{t} \sqrt{\eta} \hat{q}$ to the Hamiltonian of the system, and then taking the stochastic average $\mathbb{E}(\cdot)$. Here, $w_{t}$ is a white noise, with zero average and deltacorrelation function: $\mathbb{E}\left(w_{t} w_{s}\right)=\delta(t-s)$. Accordingly, the Hamiltonian takes the following form:

$$
\hat{H}=\frac{1}{2 m} \hat{p}^{2}+\frac{1}{2} m \omega_{0}^{2} \hat{q}^{2}-\hbar w_{t} \sqrt{\eta} \hat{q},
$$

and the corresponding Heisenberg equations of motion, where we add a term describing the coupling of the oscillator with a phononic bath, are

$$
\begin{aligned}
& \partial_{t} \hat{q}=\hat{p} / m, \\
& \partial_{t} \hat{p}=-m \omega_{0}^{2} \hat{q}+\hbar \sqrt{\eta} w_{t}-\gamma_{m} \hat{p}+\hat{\xi}(t),
\end{aligned}
$$

where the stochastic operator $\hat{\xi}(t)$ describes the Brownian motion induced by the phononic bath, and $\gamma_{m}$ is its friction constant. The autocorrelation of $\hat{\xi}(t)$, after tracing over all phononic modes, is given by Refs. [23,24] $\mathbb{E}(\langle\hat{\xi}(t) \hat{\xi}(s)\rangle)=$ $\left(\hbar m \gamma_{m} / 2 \pi\right) \int_{-\infty}^{\infty} d \omega \omega e^{-i \omega(t-s)}[\operatorname{coth}(\beta \omega)+1]$, where $\beta=$ $\hbar /\left(2 k_{B} T\right)$, with $k_{B}$ the Boltzmann constant and $T$ the temperature of the phononic bath. Notice that in the hightemperature limit, one recovers the white noise relation, i.e., $\mathbb{E}(\langle\hat{\xi}(t) \hat{\xi}(s)\rangle)=2 m \gamma_{m} k_{B} T \delta(t-s)$.

The quantity measured in the experiment is the spectrum $\mathcal{S}_{q}(\omega)$, i.e., the Fourier transform of the twotime correlation function of the oscillator's position: $\mathcal{S}_{q}(\omega)=\int_{-\infty}^{+\infty} \mathrm{d} \tau e^{-i \omega \tau} \mathbb{E}(\langle\hat{q}(t) \hat{q}(t+\tau)\rangle)$. The area under $\mathcal{S}_{q}(\omega)$ is proportional to the mean energy, or, equivalently, the temperature of the mechanical resonator. In fact, given Eq. (4), the equilibrium energy $\mathbb{E}(\langle\hat{H}\rangle)$ can be easily expressed in terms of the spectral density $\mathcal{S}_{q}(\omega)$ and $\mathcal{S}_{p}(\omega)$ of the oscillator's position and momentum [23,24]. Equation (5) gives $\hat{p}(\omega)=-i m \omega \hat{q}(\omega)$, implying that $\mathcal{S}_{p}(\omega)=m^{2} \omega^{2} \mathcal{S}_{q}(\omega)$; therefore, $\mathcal{S}_{q}(\omega)$ suffices to characterize $\mathbb{E}(\langle\hat{H}\rangle)$.

Following standard calculation $[23,24]$, we arrive at the expression $\mathbb{E}(\langle\hat{H}\rangle)=k_{B} T+\hbar^{2} Q \eta / 2 m \omega_{0}$, where $Q=$ $\omega_{0} / \gamma_{m}$ is the quality factor. One arrives at the same result also by directly solving the CSL master equation $[17,18]$. Thus, the experimental signature of CSL is a slight temperature-independent violation of the equipartition theorem. We can express the excess energy as a temperature increase:

$$
\Delta T_{\mathrm{CSL}}=\frac{\hbar^{2} Q}{2 k_{B} m \omega_{0}} \eta .
$$

We still have to estimate $\eta$, which depends on the geometry of the system and on the two phenomenological CSL parameters, as given by Eq. (3). Our experiment is based on an ultrasoft silicon cantilever, with length $L=100 \mu \mathrm{m}$, width $w=5 \mu \mathrm{m}$, and thickness $d=0.10 \mu \mathrm{m}$ [Fig. 1(a)]. A ferromagnetic microsphere based on a neodymium-iron-boron alloy (density $\varrho_{s}=7430 \mathrm{~kg} / \mathrm{m}^{3}$ ) with diameter $2 R=4.5 \mu \mathrm{m}$ is attached to the free end of the cantilever (density $\varrho_{c}=2330 \mathrm{~kg} / \mathrm{m}^{3}$ ) and is used for displacement detection, as described below. Finding $\eta$ is not straightforward, both because of the nontrivial geometry of the system, and because the motion of the cantilever is not rigid. In the Supplemental Material we show in detail how to compute $\eta$ [25]. Figure 1(b) shows the calculated CSL-induced overheating due to cantilever and microsphere and the total one, as a function of $r_{C}$, assuming the standard collapse rate $\lambda=2.2 \times 10^{-17} \mathrm{~s}^{-1}$ [2]. The cantilever contribution is significant only for $r_{C}<10^{-7} \mathrm{~m}$, while for $r_{C}>10^{-7} \mathrm{~m}$ the microsphere contribution becomes largely dominant. The larger effect of the microsphere is explained by the dependence of $\eta$ on the square of

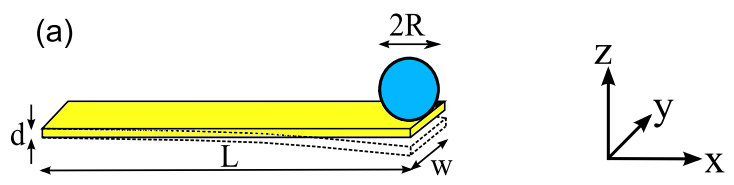

(b)

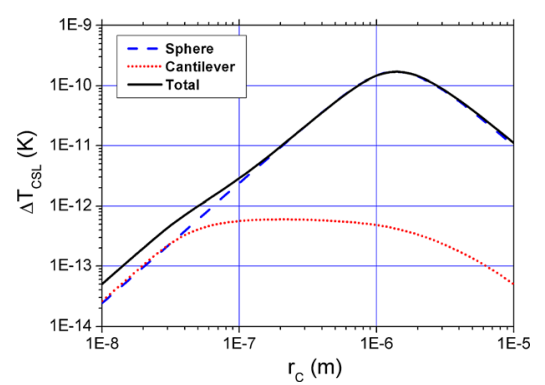

FIG. 1. (a) Scheme of the mechanical resonator. We focus on the fundamental bending mode of a high aspect ratio nanocantilever with length $L$, width $w$, and thickness $d$. A ferromagnetic microsphere with radius $R$ is attached to the free end of the cantilever. (b) Calculated CSL-induced heating $\Delta T_{\mathrm{CSL}}$ of the cantilever fundamental mode as function of $r_{C}$. The total effect (solid line) includes two terms associated, respectively, to the microsphere (dashed line)and to the cantilever (dotted line), as well as a correlation term. Because of higher density, the contribution of the sphere is largely dominant for $r_{C}>10^{-7} \mathrm{~m}$. 
the density $\varrho$. The total overheating peaks at $r_{C}=1.4 \mu \mathrm{m}$, of the order of the microsphere radius. Based on these considerations and on Eq. (6), the optimal cantilever features can be summarized as follows: size $R \sim r_{C}$, lowest possible $\omega_{0}$ and $T$, highest possible $Q$ and $\varrho_{s}$.

Experimental results. - Details on the detection scheme were already reported in Ref. [27]. A SQUID current sensor is used to detect the motion of the magnetic particle on the cantilever via a superconducting detection coil. The cantilever chip is clamped above the coil and thermalized by means of a brass spring. The cantilever-coil setup is enclosed in a superconducting shield and thermally anchored to the mixing chamber of a dilution refrigerator. The temperature is monitored by a Speer resistive thermometer, calibrated against a high-accuracy superconductive reference point device [28].

The resonant frequency of the cantilever is $f_{0}=$ $3084 \mathrm{~Hz}$ and the quality factor measured with the ringdown method is $Q=38 \times 10^{3}$. Measurements of the mean energy of the cantilever mode, or, equivalently, the effective mode temperature $T_{m}$, were performed as a function of bath temperature $T$ in the range from $10 \mathrm{mK}$ up to $1 \mathrm{~K}$. The power spectrum of the SQUID-detected signal is acquired with a resolution of $0.02 \mathrm{~Hz}$, and at least 20 spectra are averaged for each point. The spectrum is well fitted by a Lorentzian peak associated to the cantilever motion incoherently superimposed on the SQUID white noise, as seen from the examples shown in the inset of Fig. 2. An absolute calibration procedure has been developed to convert the area under the Lorentzian peak inferred from the fit into the mean energy $E$ of the cantilever mode. Details on the calibration procedure can be found in Refs. $[29,30]$.

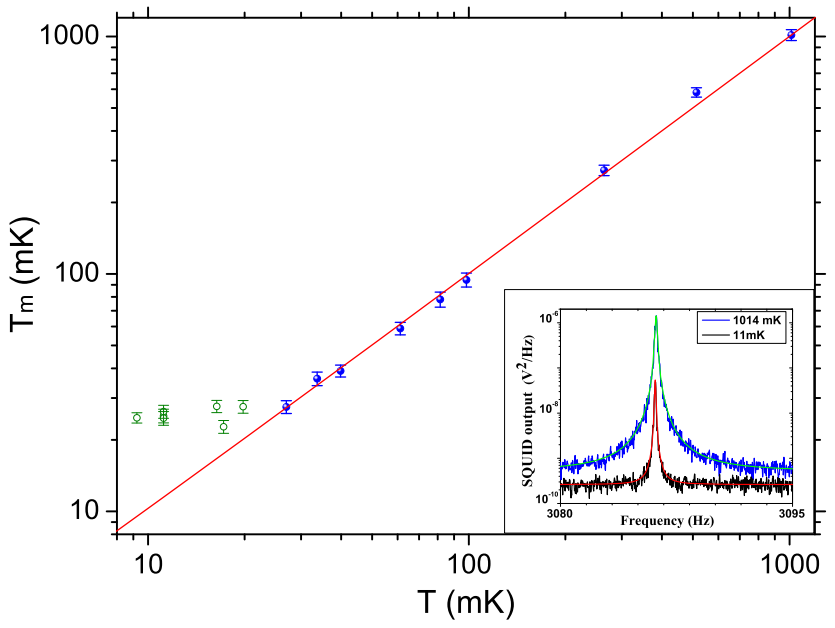

FIG. 2. Main panel: cantilever mode temperature $T_{m}=E / k_{B}$ as a function of the bath temperature $T$. Data in the saturation region $T<25 \mathrm{mK}$ are excluded by the data analysis. The straight line represents the best fit with $T_{m}=\alpha T+T_{0}$. Inset: examples of acquired averaged spectra at two representative temperatures $T=11 \mathrm{mK}$ and $T=1.01 \mathrm{~K}$, with the respective best fit with a Lorentzian curve. Figures reproduced from Ref. [27].
Figure 2 shows the measured cantilever mode temperature as a function of the bath temperature. We have divided the data set in two regions. For $T>25 \mathrm{mK}$ and up to the maximum temperature $\sim 1 \mathrm{~K}$, the data follow remarkably well the expected equipartition behavior. In particular, the parameter-free equipartition curve $T_{m}=T$ fits the experimental data well, indicating that the cantilever is well thermalized and is actually behaving as a primary thermometer. Furthermore, we can infer that the systematic errors in the calibration and in the temperature measurement are negligible within the error bar. A linear fit with variable slope $\alpha$ gives $\alpha=(1.03 \pm 0.03)$, indicating that the calibration systematic error is of the order $3 \%$ or less.

At bath temperatures lower than $25 \mathrm{mK}, T_{m}$ is found to saturate at an effective value $T_{m}=(25 \pm 1) \mathrm{mK}$. As discussed in Ref. [27], the saturation is consistent with an unknown effective heat leak to the cantilever on the order of $100 \mathrm{aW}$. The sharpness of the saturation is typical at millikelvin temperature and is caused by the strong temperature dependence of the limiting thermalization mechanisms. For instance, the heat conductivity of silicon or other thermal boundary resistances are expected to scale as $T^{n}$ with $n \sim 3$. As a consequence, the cantilever mode temperature rapidly approaches the expected linear behavior as soon as the temperature is increased above the saturation value.

The low temperature saturation cannot be attributed to CSL-induced heating, which would rather appear as a positive nonzero intercept of the measured data in the linear part. To set an upper bound on a possible CSL heating, we have to determine the maximum positive intercept consistent with the subset of experimental data following a linear behavior. To this end, we perform a linear fit of the data above $25 \mathrm{mK}$, with slope $\alpha$ fixed to 1 and the intercept $T_{0}$ as a free parameter. The fit yields $T_{0}=$ $(0.28 \pm 1.18) \mathrm{mK}$, with $\chi^{2}=1.20$. We may directly use this estimate to infer an upper limit at a given confidence level. However, one needs to be cautious when inferring upper limits on a quantity that is physically allowed to be only positive. Here, we adopt the Feldman-Cousins approach [31], which has been proposed precisely to address this kind of problems and to overcome possible misinterpretations of the confidence interval. In particular, we assume that the measured value $T_{0}$ provides an experimental estimation of the true value $\Delta T_{\mathrm{CSL}}>0$ of a positive CSL heating effect. Therefore, $T_{0}$ and $\Delta T_{\mathrm{CSL}}$ play the roles of $x$ and $\mu$ of Feldman-Cousins [31]. The standard procedure for a Gaussian-distributed $T_{0}$ provides then the upper limit $\Delta T_{\mathrm{CSL}} \leq 2.5 \mathrm{mK}$ at the 95\% confidence level.

We have also performed the same procedure starting with a linear fit with both slope and intercept as free parameters. In this case, besides the slope $\alpha=1.03 \pm 0.03$, we obtain a slightly different estimate of the intercept $T_{0}=(-1.09 \pm 1.77) \mathrm{mK}$. Nonetheless, the final upper 
limit $\Delta T_{\mathrm{CSL}} \leq 2.4 \mathrm{mK}$ at $95 \%$ confidence level is essentially unchanged.

Discussion. - Let us connect our experimental result to the CSL model. By using Eq. (6), giving the expected CSL heating, which is a function of the collapse rate $\lambda$ and the correlation length $r_{C}$, and the measured upper limit on $\Delta T_{\text {CSL }}$ discussed before, we can draw the exclusion plot shown in Fig. 3. The dashed region is excluded at the $95 \%$ confidence level.

In Fig. 3 our upper limit is compared with the best one reported so far in literature, obtained by $\mathrm{x}$-ray spontaneous emission experiments [32]. To allow for a full comparison we have extended the upper limit, reported only for $r_{C}=10^{-7} \mathrm{~m}$ in Ref. [32], to the full $r_{C}$ range. This is done by taking into account that CSL-induced $\mathrm{x}$-ray emission scales as $r_{C}^{-2}$. We have also reported the upper bound coming from matter-wave interferometry [20-22], $\lambda \leq 5.0 \times 10^{-6} \mathrm{~s}^{-1}$ for $r_{C}=10^{-7} \mathrm{~m}$. Figure 3 shows also the conservative theoretical lower bound according to the original paper of Ghirardi et al. [2] and the lower bounds suggested by Adler, based on the analysis of the latent image formation in photography [5].

At the conventional length $r_{C}=10^{-7} \mathrm{~m}$, our upper limit is still 3 orders of magnitude away from the limit set by $\mathrm{x}$-ray emission, but provides an improvement over the $\mathrm{x}$-ray

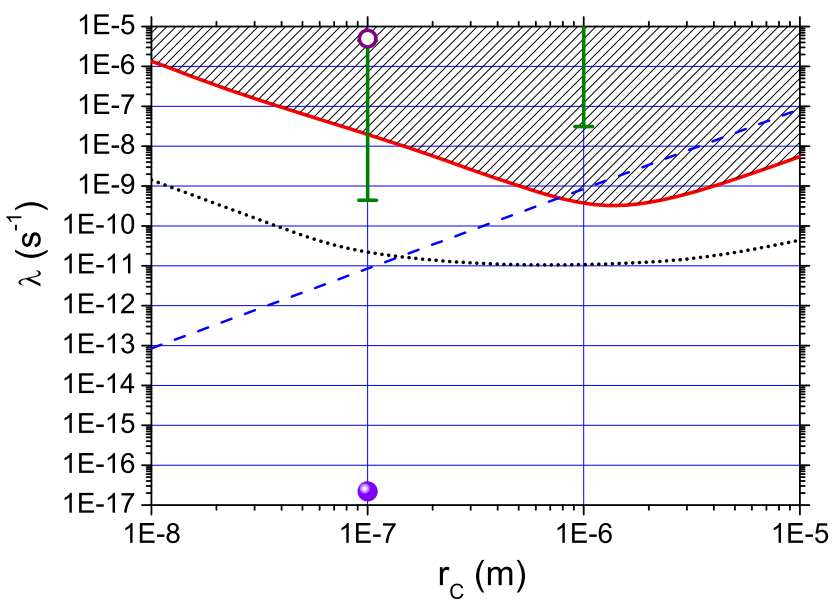

FIG. 3. Exclusion plot in the $\lambda-r_{C}$ plane based on our experimental data, compared with the best experimental upper bounds reported so far and with the proposed theoretical lower bounds. Continuous (red) curve: upper limit on the CSL collapse rate $\lambda$, as function of the characteristic length $r_{C}$. The region above the curve is excluded at $95 \%$ confidence level. Dashed (blue) curve: upper limit on $\lambda$ from spontaneous x-ray emission [32]. Dotted (black) line: foreseen upper limit from the proposed future upgraded setup (see text). Hollow (purple) circle: best upper limit on $\lambda$ from matter-wave interferometry at $r_{C}=10^{-7} \mathrm{~m}$ [20-22]. Filled (violet) circle: conservative lower bound on CSL parameters according to Ghirardi et al. [2]. Green bars: optimistic lower bounds on $\lambda$ at $r_{C}=10^{-7} \mathrm{~m}$ and $r_{C}=10^{-6} \mathrm{~m}$ as suggested by Adler [5]. limit at $r_{C}>10^{-6} \mathrm{~m}$. It also improves the bound coming from matter wave interferometry by 2 orders of magnitude.

Compared with theoretical predictions, our limit is still 9 and 7 orders of magnitude far from the conservative collapse rate proposed by Ghirardi et al. [2] at $r_{C}=10^{-7} \mathrm{~m}$ and $r_{C}=10^{-6} \mathrm{~m}$, respectively. However, it compares favorably with Adler predictions. We remark that Adler intervals are lower bounds on the CSL collapse rate. Our upper limit is thus ruling out Adler predictions completely at $r_{C} \geq 3 \times 10^{-7} \mathrm{~m}$, and partially at the conventional CSL length $r_{C}=10^{-7} \mathrm{~m}$.

Despite Adler's lower bounds are already strongly excluded by x-ray experiments, our result is still significant because of the very different time scale involved. In fact, $\mathrm{x}$-ray experiments probe the collapse field at very high frequency $\sim 10^{18} \mathrm{~Hz}$, while we probe the collapse field at low frequency $\sim 1 \mathrm{kHz}$, so that the two approaches are complementary. Moreover, it has been suggested that the limits inferred by $\mathrm{x}$-ray emission could be evaded by assuming a high frequency cutoff on the collapse field spectrum [5]. In contrast, the time scale of our experiment is comparable to that assumed by Adler when analyzing the process of latent image formation, which led to his enhanced lower bound. Therefore, our data imply that Adler's proposal is ruled out, at least for $r_{C}>3 \times 10^{-7} \mathrm{~m}$, even under the assumption of nonwhite noise.

A final point to be discussed is how our work compares with existing or foreseen experiments with mechanical resonators. Contrary to expectations and despite the stremendous recent progress, typical quantum opto- and electro-mechanical systems [33] are largely suboptimal to search for CSL heating effects, essentially because they are optimized at very high frequency $[34,35]$. The best case is likely given by MHz-frequency aluminum nanomembranes, cooled to $\sim 10 \mathrm{mK}$ by several groups [36-38]. The bound on the CSL model from these experiments is $2-3$ orders of magnitude worse than ours $[16,17]$, because of the much larger resonant frequency. Macroscopic lowfrequency resonators such as gravitational wave detectors $[39,40]$ are not effective as well, because of the very large size [16,17]. A more promising approach is given by resonators with much lower resonant frequency, such as torsion microbalances or micropendula [41] or by levitated microparticles $[18,19,42]$.

We conclude with an outlook towards future improvements of our method, achievable with existing technology. Single crystal diamond cantilevers with thickness $0.6 \mu \mathrm{m}$ have been recently demonstrated, with very high quality factors approaching $10^{7}$ at $100 \mathrm{mK}$ [43]. Combining such a device with a high density mass load (we choose as example a FePt film with size $40 \times 12 \times 0.2 \mu \mathrm{m}$ [44]) and assuming a temperature resolution $\Delta T \sim 1 \mathrm{mK}$, we obtain the dotted curve in Fig. 3. This would improve by 2-3 orders of magnitude the upper limit obtained in this work. Larger improvements towards the Ghirardi limit 
can be conceived, based on entirely new technologies $[18,19,42]$.

The experiment was financially supported by ERC and by the EU-project Microkelvin. A. V. acknowledges support from INFN. M. B. and A. B. acknowledge financial support from EU project NANOQUESTFIT, The John Templeton Foundation (Grant No. 39530), University of Trieste (FRA 2013), and INFN. We thank W. Bosch for the high-accuracy temperature calibration, M. Carlesso, C. Curceanu, K. Piscicchia, S. Donadi, H. Ulbricht, M. Paternostro, and M. Toros for useful discussions.

*andrea.mistervin@gmail.com

[1] G. C. Ghirardi, A. Rimini, and T. Weber, Phys. Rev. D 34, 470 (1986).

[2] G. C. Ghirardi, P. Pearle, and A. Rimini, Phys. Rev. A 42, 78 (1990); G. C. Ghirardi, R. Grassi, and F. Benatti, Found. Phys. 25, 5 (1995).

[3] A. Bassi and G. C. Ghirardi, Phys. Rep. 379, 257 (2003).

[4] A. Bassi, K. Lochan, S. Satin, T. P. Singh, and H. Ulbricht, Rev. Mod. Phys. 85, 471 (2013).

[5] S. L. Adler, J. Phys. A 40, 2935 (2007).

[6] K. Hornberger, S. Gerlich, P. Haslinger, S. Nimmrichter, and M. Arndt, Rev. Mod. Phys. 84, 157 (2012).

[7] T. Juffmann, H. Ulbricht, and M. Arndt, Rep. Prog. Phys. 76, 086402 (2013).

[8] M. Arndt and K. Hornberger, Nat. Phys. 10, 271 (2014).

[9] W. Marshall, C. Simon, R. Penrose, and D. Bouwmeester, Phys. Rev. Lett. 91, 130401 (2003).

[10] J. van Wezel and T. H. Oosterkamp, Proc. R. Soc. A 468, 35 (2012).

[11] O. Romero-Isart, Phys. Rev. A 84, 052121 (2011).

[12] B. Collett and P. Pearle, Found. Phys. 33, 1495 (2003).

[13] S. L. Adler, J. Phys. A 38, 2729 (2005).

[14] A. Bassi, E. Ippoliti, and S. L. Adler, Phys. Rev. Lett. 94, 030401 (2005).

[15] M. Bahrami, M. Paternostro, A. Bassi, and H. Ulbricht, Phys. Rev. Lett. 112, 210404 (2014).

[16] S. Nimmrichter, K. Hornberger, and K. Hammerer, Phys. Rev. Lett. 113, 020405 (2014).

[17] L. Diosi, Phys. Rev. Lett. 114, 050403 (2015).

[18] D. Goldwater, M. Paternostro, and P.F. Barker, arXiv: 1506.08782 .

[19] J. Li, S. Zippilli, J. Zhang, and D. Vitali, arXiv:1508.00466.

[20] S. Eibenberger, S. Gerlich, M. Arndt, M. Mayor, and J. Tüxen, Phys. Chem. Chem. Phys. 15, 14696 (2013).

[21] S. Nimmrichter, K. Hornberger, P. Haslinger, and M. Arndt, Phys. Rev. A 83, 043621 (2011).
[22] For a full analysis of CSL in matter-wave experiments, see M. Toroš and A. Bassi, arXiv:1601.03672.

[23] M. Paternostro, S. Gigan, M. S. Kim, F. Blaser, H. R. Böhm, and M. Aspelmeyer, New J. Phys. 8, 107 (2006).

[24] G. S. Agarwal, Quantum Optics (Cambridge University Press, Cambridge, England, 2012), Chap. 20.

[25] See Supplemental Material at http://link.aps.org/ supplemental/10.1103/PhysRevLett.116.090402 for detailed calculations, which includes Ref. [26].

[26] A. Erturk and D. J. Inman, Piezoelectric Energy Harvesting (John Wiley \& Sons, New York, 2011), Appendix C.

[27] O. Usenko, A. Vinante, G. Wijts, and T. H. Oosterkamp, Appl. Phys. Lett. 98, 133105 (2011).

[28] SRD1000 Measurement Systems (HDL, Netherlands, http:// hdleiden.home.xs4all.nl/srd1000).

[29] See Supplemental Material of Ref. [27].

[30] O. Usenko, Ph.D Thesis, Leiden University, Netherlands, 2012.

[31] G. J. Feldman and R. D. Cousins, Phys. Rev. D 57, 3873 (1998).

[32] C. Curceanu, B. C. Hiesmayr, and K. Piscicchia, J. Adv. Phys. 4, 263 (2015).

[33] M. Aspelmeyer, T. Kippenberg, and F. Marquardt, Rev. Mod. Phys. 86, 1391 (2014).

[34] A. D. O’Connell et al., Nature (London) 464, 697 (2010).

[35] J. Chan, T. P. Mayer Alegre, A. H. Safavi-Naeini, J. T. Hill, A. Krause, S. Gröblacher, M. Aspelmeyer, and O. Painter, Nature (London) 478, 89 (2011).

[36] J. D. Teufel, T. Donner, D. Li, J. H. Harlow, M. S. Allman, K. Cicak, A. J. Sirois, J. D. Whittaker, K. W. Lehnert, and R. W. Simmonds, Nature (London) 475, 359 (2011).

[37] E. E. Wollman, C. U. Lei, A. J. Weinstein, J. Suh, A. Kronwald, F. Marquardt, A. A. Clerk, and K. C. Schwab, Science 349, 952 (2015).

[38] J.-M. Pirkkalainen, E. Damskägg, M. Brandt, F. Massel, and M. A. Sillanpää, Phys. Rev. Lett. 115, 243601 (2015).

[39] A. Vinante (for the AURIGA Collaboration), Classical Quantum Gravity 23, S103 (2006).

[40] J. Aasi et al. LIGO Scientific Collaboration, Classical Quantum Gravity 32, 074001 (2015).

[41] N. Matsumoto, K. Komori, Y. Michimura, G. Hayase, Y. Aso, and K. Tsubono, Phys. Rev. A 92, 033825 (2015).

[42] O. Romero-Isart, L. Clemente, C. Navau, A. Sanchez, and J. I. Cirac, Phys. Rev. Lett. 109, 147205 (2012).

[43] Y. Tao, J. M. Boss, B. A. Moores, and C. L. Degen, Nat. Commun. 5, 3638 (2014).

[44] H. C. Overweg, A. M. J. den Haan, H. J. Eerkens, P. F. A. Alkemade, A. L. La Rooij, R. J. C. Spreeuw, L. Bossoni, and T. H. Oosterkamp, Appl. Phys. Lett. 107, 072402 (2015). 